\title{
ङु \\ Theory of spin Hall magnetoresistance
}

\author{
Yan-Ting Chen, ${ }^{1}$ Saburo Takahashi, ${ }^{2}$ Hiroyasu Nakayama, ${ }^{2}$ Matthias Althammer, ${ }^{3,4}$ Sebastian T. B. Goennenwein, ${ }^{3}$ \\ Eiji Saitoh, 2,5,6,7 and Gerrit E. W. Bauer ${ }^{1,2,5}$ \\ ${ }^{1}$ Kavli Institute of NanoScience, Delft University of Technology, Lorentzweg 1, 2628 CJ Delft, The Netherlands \\ ${ }^{2}$ Institute for Materials Research, Tohoku University, Sendai 980-8577, Japan \\ ${ }^{3}$ Walther-Meißner-Institut, Bayerische Akademie der Wissenschaften, 85748 Garching, Germany \\ ${ }^{4}$ Center for Materials for Information Technology MINT, Department of Chemistry, University of Alabama, Tuscaloosa, Alabama 35487, USA \\ ${ }^{5}$ WPI Advanced Institute for Materials Research, Tohoku University, Sendai 980-8577, Japan \\ ${ }^{6}$ CREST, Japan Science and Technology Agency, Tokyo 102-0076, Japan \\ ${ }^{7}$ The Advanced Science Research Center, Japan Atomic Energy Agency, Tokai 319-1195, Japan
}

(Received 7 February 2013; published 12 April 2013)

\begin{abstract}
We present a theory of the spin Hall magnetoresistance (SMR) in multilayers made from an insulating ferromagnet $\mathrm{F}$, such as yttrium iron garnet (YIG), and a normal metal $\mathrm{N}$ with spin-orbit interactions, such as platinum $(\mathrm{Pt})$. The SMR is induced by the simultaneous action of spin Hall and inverse spin Hall effects and therefore a nonequilibrium proximity phenomenon. We compute the SMR in $\mathrm{F} \mid \mathrm{N}$ and $\mathrm{F}|\mathrm{N}| \mathrm{F}$ layered systems, treating $\mathrm{N}$ by spin-diffusion theory with quantum mechanical boundary conditions at the interfaces in terms of the spin-mixing conductance. Our results explain the experimentally observed spin Hall magnetoresistance in $\mathrm{N} \mid \mathrm{F}$ bilayers. For $\mathrm{F}|\mathrm{N}| \mathrm{F}$ spin valves we predict an enhanced SMR amplitude when magnetizations are collinear. The SMR and the spin-transfer torques in these trilayers can be controlled by the magnetic configuration.
\end{abstract}

DOI: 10.1103/PhysRevB.87.144411 PACS number(s): 85.75.-d, 72.15.Gd, 73.43.Qt, 72.25.Mk

\section{INTRODUCTION}

Spin currents are a central theme in spintronics since they are intimately associated with the manipulation and transport of spins in small structures and devices. ${ }^{1,2}$ Spin currents can be generated by means of the spin Hall effect (SHE) and detected by the inverse spin Hall effect (ISHE). ${ }^{3}$ Of special interest are multilayers made of normal metals $(\mathrm{N})$ and ferromagnets $(\mathrm{F})$. When an electric current flows through $\mathrm{N}$, an SHE spin current flows towards the interfaces, where it can be absorbed as a spin-transfer torque (STT) on the ferromagnet. This STT affects the magnetization damping ${ }^{4}$ or even switches the magnetization. 5,6 The ISHE can be used to detect spin currents pumped by the magnetization dynamics excited by microwaves ${ }^{7-10}$ or temperature gradients (spin Seebeck effect). ${ }^{11,12}$

Recently, magnetic insulators have attracted the attention of the spintronics community. Yttrium iron garnets (YIG), a class of ferrimagnetic insulators with a large band gap, are interesting because of their very low magnetization damping. Their magnetization can be activated thermally to generate the spin Seebeck effect in YIG|Pt bilayers. ${ }^{13,14}$ By means of the SHE, spin waves can be electrically excited in YIG via a Pt contact, and, via the ISHE, subsequently detected electrically in another Pt contact. ${ }^{15}$ Spin transport at an $\mathrm{N} / \mathrm{F}$ interface is governed by the complex spin-mixing conductance $G_{\uparrow \downarrow} \cdot{ }^{16}$ The prediction of a large real part of $G_{\uparrow \downarrow}$ for interfaces of YIG with simple metals by first-principles calculations ${ }^{17}$ has been confirmed by experiments. ${ }^{18}$

Magnetoresistance (MR) is the property of a material to change the value of its electrical resistance under an external magnetic field. In normal metals its origin is the Lorentz force. ${ }^{19}$ The dependence of the resistance on the angle between current and magnetization in metallic ferromagnets is called anisotropic magnetoresistance (AMR). The transverse component of the AMR is also called the planar Hall effect
(PHE), i.e., the transverse (Hall) voltage found in ferromagnets when the magnetization is rotated in the plane of the film. ${ }^{20,21}$ Both effects are symmetric with respect to magnetization reversal, which distinguishes them from the anomalous Hall effect (AHE) for magnetizations normal to the film, which changes sign under magnetization reversal. ${ }^{22}$ The physical origin of AMR, PHE, and AHE is the spin-orbit interaction, in contrast to the giant magnetoresistance (GMR), which reflects the change in resistance that accompanies the magnetic-fieldinduced magnetic configuration in magnetic multilayers. ${ }^{23}$

Here we propose a theory for a recently discovered magnetoresistance effect in Pt|YIG bilayer systems. ${ }^{14,24-26}$ This MR is remarkable since YIG is a very good electric insulator such that a charge current can only flow in Pt. We explain this unusual magnetoresistance not in terms of an equilibrium static magnetic proximity polarization in $\mathrm{Pt}^{24}$ but rather in terms of a nonequilibrium proximity effect caused by the simultaneous action of the SHE and ISHE and therefore call it spin Hall magnetoresistance (SMR). This effect scales like the square of the spin Hall angle and is modulated by the magnetization direction in YIG via the spin transfer at the $\mathrm{N} / \mathrm{F}$ interface. Our explanation is similar to the Hanle-effectinduced magnetoresistance in the two-dimensional electron gas proposed by Dyakonov. ${ }^{27}$ Here we present the details of our theory, which is based on the spin-diffusion approximation in the $\mathrm{N}$ layer in the presence of spin-orbit interactions $\mathrm{s}^{28}$ and quantum mechanical boundary conditions at the interface in terms of the spin-mixing conductance. ${ }^{16,17} \mathrm{We}$ also address $\mathrm{F}|\mathrm{N}| \mathrm{F}$ spin valves with electric currents applied parallel to the interface(s) with the additional degree of freedom of the relative angle between the two magnetization directions.

This paper is organized as follows. We present the model, i.e., spin diffusion with proper boundary conditions, in Sec. II. In Sec. III, we consider an $\mathrm{N} \mid \mathrm{F}$ bilayer as shown in Fig. 1(a). We obtain spin accumulation, spin currents, and finally the measured charge currents that are compared with 

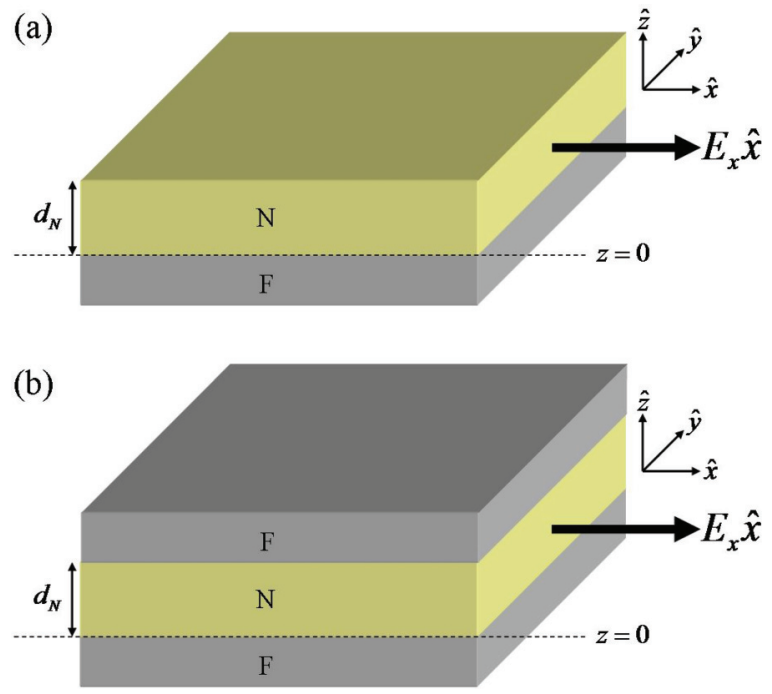

FIG. 1. (Color online) (a) $\mathrm{N} \mid \mathrm{F}$ bilayer and (b) $\mathrm{F}|\mathrm{N}| \mathrm{F}$ trilayer systems considered here, where $\mathrm{F}$ is a ferromagnetic insulator and $\mathrm{N}$ a normal metal.

the experimental SMR. We also find and discuss that the imaginary part of the spin-mixing conductance generates an AHE. F|N|F [Fig. 1(b)] spin valves are investigated in Sec. IV, which show an enhanced SMR for spacers thinner than the spin-flip diffusion length. We summarize the results and give conclusions in Sec. V.

\section{TRANSPORT THEORY IN METALS IN CONTACT WITH A MAGNETIC INSULATOR}

The spin current density in the nonrelativistic limit

$$
\overleftrightarrow{\mathbf{j}_{s}}=e n\langle\vec{v} \otimes \overrightarrow{\boldsymbol{\sigma}}+\overrightarrow{\boldsymbol{\sigma}} \otimes \vec{v}\rangle / 2=\left(\vec{j}_{s x}, \vec{j}_{s y}, \vec{j}_{s z}\right)^{T}=\left(\vec{j}_{s}^{x}, \vec{j}_{s}^{y}, \vec{j}_{s}^{z}\right)
$$

is a second-order tensor (in units of the charge current density $\left.\vec{j}_{c}=e n\langle\vec{v}\rangle\right)$, where $e=|e|$ is the electron charge, $n$ is the density of the electrons, $\vec{v}$ is the velocity operator, $\vec{\sigma}$ is the vector of Pauli spin matrices, and $\langle\cdots\rangle$ denotes an expectation value. The row vectors $\vec{j}_{s i}=e n\left\langle\vec{v} \sigma_{i}+\sigma_{i} \vec{v}\right\rangle / 2$ in Eq. (1) are the spin current densities polarized in the $\hat{\imath}$ direction, while the column vectors $\vec{j}_{s}^{j}=e n\left\langle v_{j} \overrightarrow{\boldsymbol{\sigma}}+\overrightarrow{\boldsymbol{\sigma}} v_{j}\right\rangle / 2$ denote the spin current densities with polarization $\overrightarrow{\boldsymbol{\sigma}}$ flowing in the $\hat{\jmath}$ direction. Ohm's law for metals with spin-orbit interactions can be summarized by the relation between thermodynamic driving forces and currents that reflects Onsager's reciprocity by the symmetry of the response matrix: ${ }^{28}$

$$
\begin{aligned}
\left(\begin{array}{c}
\vec{j}_{c} \\
\vec{j}_{s x} \\
\vec{j}_{s y} \\
\vec{j}_{s z}
\end{array}\right)= & \left(\begin{array}{cccc}
1 & \theta_{\mathrm{SH}} \hat{x} \times & \theta_{\mathrm{SH}} \hat{y} \times & \theta_{\mathrm{SH}} \hat{z} \times \\
\theta_{\mathrm{SH}} \hat{x} \times & 1 & 0 & 0 \\
\theta_{\mathrm{SH}} \hat{y} \times & 0 & 1 & 0 \\
\theta_{\mathrm{SH}} \hat{z} \times & 0 & 0 & 1
\end{array}\right) \\
& \left(\begin{array}{c}
-\vec{\nabla} \mu_{0} / e \\
-\vec{\nabla} \mu_{s x} /(2 e) \\
-\vec{\nabla} \mu_{s y} /(2 e) \\
-\vec{\nabla} \mu_{s z} /(2 e)
\end{array}\right),
\end{aligned}
$$

where $\vec{\mu}_{s}=\left(\mu_{s x}, \mu_{s y}, \mu_{s z}\right)^{T}-\mu_{0} \hat{1}$ is the spin accumulation, i.e., the spin-dependent chemical potential relative to the charge chemical potential $\mu_{0}, \sigma$ is the electric conductivity, $\theta_{\mathrm{SH}}$ is the spin Hall angle, and " $x$ " denotes the vector cross product operating on the gradients of the spin-dependent chemical potentials. The spin Hall effect is represented by the lower nondiagonal elements that generate the spin currents in the presence of an applied electric field, in the following chosen to be in the $\hat{x}$ direction $\vec{E}=E_{x} \hat{x}=-\hat{x} \partial_{x} \mu_{0} / e$. The inverse spin Hall effect is governed by elements above the diagonal that connect the gradients of the spin accumulations to the charge current density.

The spin accumulation $\vec{\mu}_{s}$ is obtained from the spindiffusion equation in the normal metal

$$
\nabla^{2} \vec{\mu}_{s}=\frac{\vec{\mu}_{s}}{\lambda^{2}}
$$

where the spin-diffusion length $\lambda=\sqrt{D \tau_{\mathrm{sf}}}$ is expressed in terms of the charge diffusion constant $D$ and spin-flip relaxation time $\tau_{\mathrm{sf}}{ }^{29}$ For films with thickness $d_{N}$ in the $\hat{z}$ direction,

$$
\vec{\mu}_{s}(z)=\vec{A} e^{-z / \lambda}+\vec{B} e^{z / \lambda},
$$

where the constant column vectors $\vec{A}$ and $\vec{B}$ are determined by the boundary conditions at the interfaces.

According to Eq. (2), the spin current in $\mathrm{N}$ consists of diffusion and spin Hall drift contributions. Since we are considering a system homogeneous in the $x-y$ plane, we focus on the spin current density flowing in the $\hat{z}$ direction

$$
\vec{j}_{s}^{z}(z)=-\frac{\sigma}{2 e} \partial_{z} \vec{\mu}_{s}-j_{s 0}^{\mathrm{SH}} \hat{y},
$$

where $j_{s 0}^{\mathrm{SH}}=\theta_{\mathrm{SH}} \sigma E_{x}$ is the bare spin Hall current, i.e., the spin current generated directly by the SHE.

The boundary conditions require that $\vec{j}_{s}^{z}(z)$ is continuous at the interfaces $z=d_{N}$ and $z=0$. The spin current at a vacuum (V) interface vanishes, $\vec{j}_{s}^{(\mathrm{V})}=0$. The spin current density $\vec{j}_{s}^{(\mathrm{F})}$ at a magnetic interface is governed by the spin accumulation and spin-mixing conductance: ${ }^{16}$

$$
e \vec{j}_{s}^{(\mathrm{F})}(\hat{m})=G_{r} \hat{m} \times\left(\hat{m} \times \vec{\mu}_{s}\right)+G_{i}\left(\hat{m} \times \vec{\mu}_{s}\right),
$$

where $\hat{m}=\left(m_{x}, m_{y}, m_{z}\right)^{T}$ is a unit vector along the magnetization and $G_{\uparrow \downarrow}=G_{r}+i G_{i}$ the complex spin-mixing interface conductance per unit area. The imaginary part $G_{i}$ can be interpreted as an effective exchange field acting on the spin accumulation. A positive current in Eq. (6) corresponds to up spins flowing from $\mathrm{F}$ towards N. Since F is an insulator, this spin current density is proportional to the spin transfer torque acting on the ferromagnet

$$
\vec{\tau}_{\text {STT }}=-\frac{\hbar}{2 e} \hat{m} \times\left(\hat{m} \times \vec{j}_{s}^{(\mathrm{F})}\right)=\frac{\hbar}{2 e} \vec{j}_{s}^{(\mathrm{F})} .
$$

With these boundary conditions we determine the coefficients $\vec{A}$ and $\vec{B}$, which leads to the spin accumulation

$$
\begin{aligned}
\vec{\mu}_{s}= & \frac{2 e \lambda}{\sigma}\left[-\left(j_{s 0}^{\mathrm{SH}} \hat{y}+\vec{j}_{s}^{z}\left(d_{N}\right)\right) \cosh \frac{z}{\lambda}\right. \\
& \left.+\left(j_{s 0}^{\mathrm{SH}} \hat{y}+\vec{j}_{s}^{(\mathrm{F})}(\hat{m})\right) \cosh \frac{z-d_{N}}{\lambda}\right] / \sinh \frac{d_{N}}{\lambda},
\end{aligned}
$$


where $\vec{j}_{s}^{z}\left(d_{N}\right)=0$ for $\mathrm{F}(\hat{m})|\mathrm{N}| \mathrm{V}$ bilayers and $\vec{j}_{s}^{z}\left(d_{N}\right)=$ $-\vec{j}_{s}^{(\mathrm{F})}\left(\hat{m}^{\prime}\right)$ for $\mathrm{F}(\hat{m})|\mathrm{N}| \mathrm{F}\left(\hat{m}^{\prime}\right)$ spin valves.

\section{N|F BILAYERS}

In the bilayer the spin accumulation (8) is

$$
\vec{\mu}_{s}(z)=-\hat{y} \mu_{s}^{0} \frac{\sinh \frac{2 z-d_{N}}{2 \lambda}}{\sinh \frac{d_{N}}{2 \lambda}}+\vec{j}_{s}^{(\mathrm{F})}(\hat{m}) \frac{2 e \lambda}{\sigma} \frac{\cosh \frac{z-d_{N}}{\lambda}}{\sinh \frac{d_{N}}{\lambda}},
$$

where $\mu_{s}^{0} \equiv\left|\vec{\mu}_{s}(0)\right|=(2 e \lambda / \sigma) j_{s 0}^{\mathrm{SH}} \tanh \left[d_{N} /(2 \lambda)\right]$ is the spin accumulation at the interface in the absence of spin transfer, i.e., when $G_{\uparrow \downarrow}=0$.

Using Eq. (6), the spin accumulation at $z=0$ becomes

$$
\begin{aligned}
\vec{\mu}_{s}(0)= & \hat{y} \mu_{s}^{0}+\frac{2 \lambda}{\sigma}\left[G_{r}\left\{\hat{m}\left[\hat{m} \cdot \vec{\mu}_{s}(0)\right]-\vec{\mu}_{s}(0)\right\}\right. \\
& \left.+G_{i} \hat{m} \times \vec{\mu}_{s}(0)\right] \operatorname{coth} \frac{d_{N}}{\lambda} .
\end{aligned}
$$

With

$$
\begin{gathered}
\hat{m} \cdot \vec{\mu}_{s}(0)=m_{y} \mu_{s}^{0}, \\
\hat{m} \times \vec{\mu}_{s}(0)=\mu_{s}^{0} \frac{\sigma \hat{m} \times \hat{y}+\hat{m} m_{y} 2 \lambda G_{i} \operatorname{coth} \frac{d_{N}}{\lambda}}{\sigma+2 \lambda G_{r} \operatorname{coth} \frac{d_{N}}{\lambda}}-\vec{\mu}_{s}(0) \frac{2 \lambda G_{i} \operatorname{coth} \frac{d_{N}}{\lambda}}{\sigma+2 \lambda G_{r} \operatorname{coth} \frac{d_{N}}{\lambda}}, \\
\vec{\mu}_{s}(0)=\hat{y} \mu_{s}^{0} \frac{\frac{2 \lambda}{\sigma} G_{r} \operatorname{coth} \frac{d_{N}}{\lambda}\left(1+\frac{2 \lambda}{\sigma} G_{r} \operatorname{coth} \frac{d_{N}}{\lambda}\right)+\left(\frac{2 \lambda}{\sigma} G_{i} \operatorname{coth} \frac{d_{N}}{\lambda}\right)^{2}}{\left(1+\frac{2 \lambda}{\sigma} G_{r} \operatorname{coth} \frac{d_{N}}{\lambda}\right.} \\
+(\hat{m} \times \hat{y}) \mu_{s}^{0} \frac{\left.\frac{2 \lambda}{\sigma} G_{r} \operatorname{coth} \frac{d_{N}}{\lambda}\right)^{2}+\left(\frac{2 \lambda}{\sigma} G_{i} \operatorname{coth} \frac{d_{N}}{\lambda}\right)^{2}+\left(\frac{2 \lambda}{\sigma} G_{i} \operatorname{coth} \frac{d_{N}}{\lambda}\right)^{2}}{\left(1+\frac{2 \lambda}{\sigma} G_{r} \operatorname{coth} \frac{d_{N}}{\lambda}\right)^{2}+\left(\frac{2 \lambda}{\sigma} G_{i} \operatorname{coth} \frac{d_{N}}{\lambda}\right)^{2}},
\end{gathered}
$$

the spin current through the $\mathrm{F} \mid \mathrm{N}$ interface reads

$$
\vec{j}_{s}^{(\mathrm{F})}=\frac{\mu_{s}^{0}}{e} \hat{m} \times(\hat{m} \times \hat{y}) \sigma \operatorname{Re} \frac{G_{\uparrow \downarrow}}{\sigma+2 \lambda G_{\uparrow \downarrow} \operatorname{coth} \frac{d_{N}}{\lambda}}+\frac{\mu_{s}^{0}}{e}(\hat{m} \times \hat{y}) \sigma \operatorname{Im} \frac{G_{\uparrow \downarrow}}{\sigma+2 \lambda G_{\uparrow \downarrow} \operatorname{coth} \frac{d_{N}}{\lambda}} .
$$

The spin accumulation

$$
\frac{\vec{\mu}_{S}(z)}{\mu_{s}^{0}}=-\hat{y} \frac{\sinh \frac{2 z-d_{N}}{2 \lambda}}{\sinh \frac{d_{N}}{2 \lambda}}+[\hat{m} \times(\hat{m} \times \hat{y}) \operatorname{Re}+(\hat{m} \times \hat{y}) \operatorname{Im}] \frac{2 \lambda G_{\uparrow \downarrow}}{\sigma+2 \lambda G_{\uparrow \downarrow} \operatorname{coth} \frac{d_{N}}{\lambda}} \frac{\cosh \frac{z-d_{N}}{\lambda}}{\sinh \frac{d_{N}}{\lambda}}
$$

then leads to the distributed spin current in $\mathrm{N}$

$$
\frac{\vec{j}_{s}^{z}(z)}{j_{s 0}^{\mathrm{SH}}}=\hat{y} \frac{\cosh \frac{2 z-d_{N}}{2 \lambda}-\cosh \frac{d_{N}}{2 \lambda}}{\cosh \frac{d_{N}}{2 \lambda}}-[\hat{m} \times(\hat{m} \times \hat{y}) \operatorname{Re}+(\hat{m} \times \hat{y}) \operatorname{Im}] \frac{2 \lambda G_{\uparrow \downarrow} \tanh \frac{d_{N}}{2 \lambda}}{\sigma+2 \lambda G_{\uparrow \downarrow} \operatorname{coth} \frac{d_{N}}{\lambda}} \frac{\sinh \frac{z-d_{N}}{\lambda}}{\sinh \frac{d_{N}}{\lambda}} .
$$

The ISHE drives a charge current in the $x$ - $y$ plane by the diffusion spin current component flowing along the $\hat{z}$ direction. The total longitudinal (along $\hat{x}$ ) and transverse or Hall (along $\hat{y}$ ) charge currents become

$$
\begin{gathered}
\frac{j_{c, \text { long }}(z)}{j_{c}^{0}}=1+\theta_{\mathrm{SH}}^{2}\left[\frac{\cosh \frac{2 z-d_{N}}{2 \lambda}}{\cosh \frac{d_{N}}{2 \lambda}}+\left(1-m_{y}^{2}\right) \operatorname{Re} \frac{2 \lambda G_{\uparrow \downarrow} \tanh \frac{d_{N}}{2 \lambda}}{\sigma+2 \lambda G_{\uparrow \downarrow} \operatorname{coth} \frac{d_{N}}{\lambda}} \frac{\sinh \frac{z-d_{N}}{\lambda}}{\sinh \frac{d_{N}}{\lambda}}\right], \\
\frac{j_{c \text {,trans }}(z)}{j_{c}^{0}}=\theta_{\mathrm{SH}}^{2}\left(m_{x} m_{y} \operatorname{Re}-m_{z} \operatorname{Im}\right) \frac{2 \lambda G_{\uparrow \downarrow} \tanh \frac{d_{N}}{2 \lambda}}{\sigma+2 \lambda G_{\uparrow \downarrow} \operatorname{coth} \frac{d_{N}}{\lambda}} \frac{\sinh \frac{z-d_{N}}{\lambda}}{\sinh \frac{d_{N}}{\lambda}}
\end{gathered}
$$

where $j_{c}^{0}=\sigma E_{x}$ is the charge current driven by the external electric field.

The charge current vector is the observable in the experiment that is usually expressed in terms of the longitudinal and transverse (Hall) resistivities. Averaging the electric currents over the film thickness $z$ and expanding the longitudinal resistivity governed by the current in the $(x)$ direction of the applied field to leading order in $\theta_{\mathrm{SH}}^{2}$, we obtain

$$
\begin{gathered}
\rho_{\text {long }}=\sigma_{\text {long }}^{-1}=\left(\frac{\overline{j_{c, \text { long }}}}{E_{x}}\right)^{-1} \approx \rho+\Delta \rho_{0}+\Delta \rho_{1}\left(1-m_{y}^{2}\right), \\
\rho_{\text {trans }}=-\frac{\sigma_{\text {trans }}}{\sigma_{\text {long }}^{2}} \approx-\frac{\overline{j_{c \text { trans }}} / E_{x}}{\sigma^{2}}=\Delta \rho_{1} m_{x} m_{y}+\Delta \rho_{2} m_{z},
\end{gathered}
$$

where

$$
\begin{gathered}
\frac{\Delta \rho_{0}}{\rho}=-\theta_{\mathrm{SH}}^{2} \frac{2 \lambda}{d_{N}} \tanh \frac{d_{N}}{2 \lambda}, \\
\frac{\Delta \rho_{1}}{\rho}=\theta_{\mathrm{SH}}^{2} \frac{\lambda}{d_{N}} \operatorname{Re} \frac{2 \lambda G_{\uparrow \downarrow} \tanh ^{2} \frac{d_{N}}{2 \lambda}}{\sigma+2 \lambda G_{\uparrow \downarrow} \operatorname{coth} \frac{d_{N}}{\lambda}}, \\
\frac{\Delta \rho_{2}}{\rho}=-\theta_{\mathrm{SH}}^{2} \frac{\lambda}{d_{N}} \operatorname{Im} \frac{2 \lambda G_{\uparrow \downarrow} \tanh ^{2} \frac{d_{N}}{2 \lambda}}{\sigma+2 \lambda G_{\uparrow \downarrow} \operatorname{coth} \frac{d_{N}}{\lambda}},
\end{gathered}
$$

and $\rho=\sigma^{-1}$ is the intrinsic electric resistivity of the bulk normal metal. $\Delta \rho_{0}<0$ seems to imply that the resistivity is reduced by the spin-orbit interaction. However, this is an effect of the order of $\theta_{\mathrm{SH}}^{2}$ that becomes relevant only when $d_{N}$ is 
sufficiently small. The spin-orbit interaction also generates spin-flip scattering that increases the resistance to leading order according to Matthiesen's rule. We see that $\Delta \rho_{1}$ (caused mainly by $G_{r}$ ) contributes to the conductance modulation that depends on the in-plane component of the magnetization, while $\Delta \rho_{2}$ (caused mainly by $G_{i}$ ) contributes only when there is a magnetization component normal to the plane (AHE), as discussed below.

\section{A. Limit of $G_{i}=\operatorname{Im} G_{\uparrow \downarrow} \ll \operatorname{Re} G_{\uparrow \downarrow}=G_{r}$}

According to first-principles calculations, ${ }^{17}\left|G_{i}\right|$ is at least one order of magnitude smaller than $G_{r}$ for YIG, so $G_{i}=0$ appears to be a good first approximation. In this limit, we plot normalized components of spin accumulation $\left(\mu_{s x}\right.$ and $\left.\mu_{s y}\right)$ and spin current $\left(j_{s x}=\vec{j}_{s}^{z} \cdot \hat{x}\right.$ and $\left.j_{s y}=\vec{j}_{s}^{z} \cdot \hat{y}\right)$ as functions of $z$ for different magnetizations in Fig. 2. When the magnetization of $\mathrm{F}$ is along $\hat{y}$, the spin current at the $\mathrm{N} \mid \mathrm{F}$ interface $(z=0)$ vanishes just as for the vacuum interface. By rotating the magnetization from $\hat{y}$ to $\hat{x}$, the spin current at the $\mathrm{N} \mid \mathrm{F}$ interface and the torque on the magnetization is activated, while the spin accumulation is dissipated correspondingly. We note that the $x$ components of both spin accumulation and spin current vanish when the magnetization is along $\hat{x}$ and $\hat{y}$, and reach a maximum value for $\hat{m}=(\hat{x}+\hat{y}) / \sqrt{2}$.

For $G_{i}=0$ the observable transport properties reduce to

$$
\begin{gathered}
\rho_{\text {long }} \approx \rho+\Delta \rho_{0}+\Delta \rho_{1}\left(1-m_{y}^{2}\right), \\
\rho_{\text {trans }} \approx \Delta \rho_{1} m_{x} m_{y},
\end{gathered}
$$

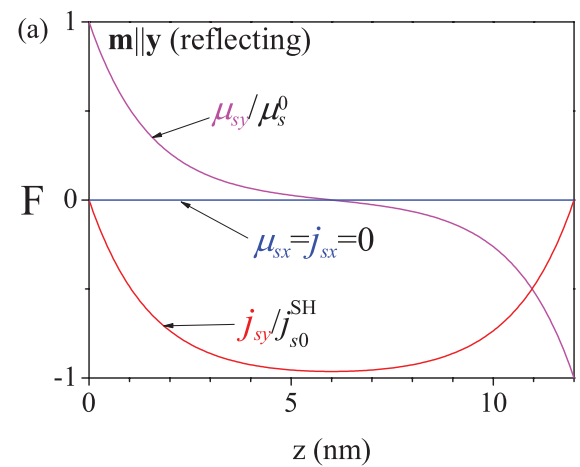

where

$$
\begin{gathered}
\frac{\Delta \rho_{0}}{\rho}=-\theta_{\mathrm{SH}}^{2} \frac{2 \lambda}{d_{N}} \tanh \frac{d_{N}}{2 \lambda}, \\
\frac{\Delta \rho_{1}}{\rho}=\theta_{\mathrm{SH}}^{2} \frac{\lambda}{d_{N}} \frac{2 \lambda G_{r} \tanh ^{2} \frac{d_{N}}{2 \lambda}}{\sigma+2 \lambda G_{r} \operatorname{coth} \frac{d_{N}}{\lambda}} .
\end{gathered}
$$

Equations (24) and (25) fully explain the magnetization dependence of SMR in Ref. 25, while Eq. (27) shows that an SMR exists only when the spin-mixing conductance does not vanish. Since results do not depend on the $z$ component of magnetization, the AHE analogue vanishes in our model when $G_{i}=0$.

\section{B. $G_{r} \gg \sigma /(2 \lambda)$}

Here we discuss the limit in which the spin current transverse to $\hat{m}$ is completely absorbed as an STT without reflection. This ideal situation is actually not so far from reality for the recently found large $G_{r}$ between YIG and noble metals. ${ }^{17,18}$ The spin current at the interface is then

$$
\frac{\vec{j}_{S}^{(\mathrm{F})}}{j_{s 0}^{\mathrm{SH}}} \stackrel{G_{r} \gg \sigma /(2 \lambda)}{=} \hat{m} \times(\hat{m} \times \hat{y}) \tanh \frac{d_{N}}{\lambda} \tanh \frac{d_{N}}{2 \lambda},
$$

and the maximum magnetoresistance for the bilayer is

$$
\frac{\Delta \rho_{1}}{\rho}=\theta_{\mathrm{SH}}^{2} \frac{\lambda}{d_{N}} \tanh \frac{d_{N}}{\lambda} \tanh ^{2} \frac{d_{N}}{2 \lambda} .
$$

In Sec. III E we test this limit with available parameters from experiments.

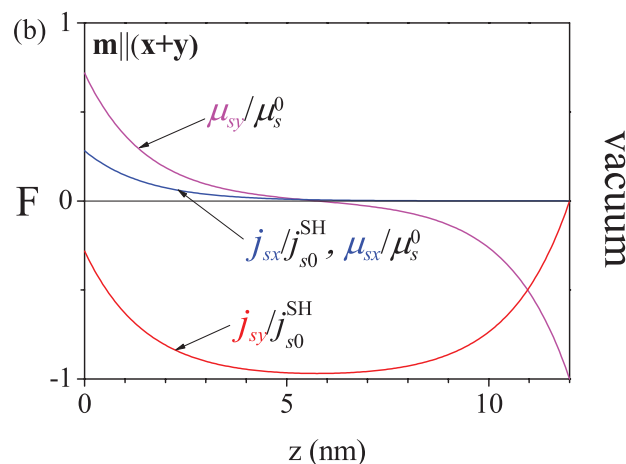

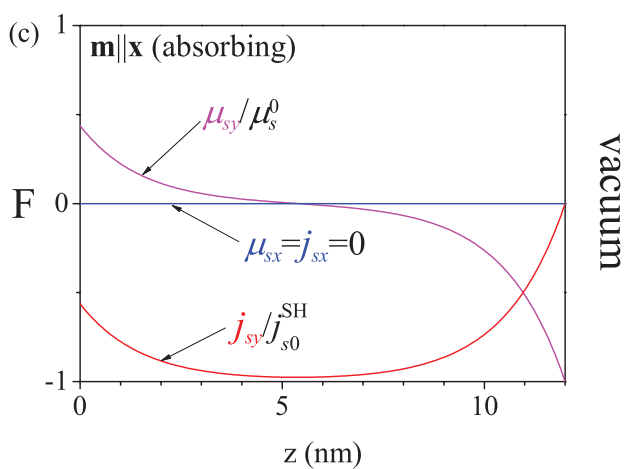

FIG. 2. (Color online) Normalized $\mu_{s x}, \mu_{s y}, j_{s x}$, and $j_{s y}$ as functions of $z$ for magnetizations (a) $\hat{m}=\hat{y}$, (b) $\hat{m}=(\hat{x}+\hat{y}) / \sqrt{2}$, and (c) $\hat{m}=\hat{x}$ for a sample with $d_{N}=12 \mathrm{~nm}$. We adopt the transport parameters $\rho=8.6 \times 10^{-7} \Omega \mathrm{m}, \lambda=1.5 \mathrm{~nm}$, and $G_{r}=5 \times 10^{14} \Omega^{-1} \mathrm{~m}^{-2}$. For magnetizations $\hat{m}=\hat{y}$ and $\hat{m}=\hat{x}$, both $\mu_{s x}$ and $j_{s x}$ vanish. 


\section{C. $\lambda / d_{N} \gg 1$}

When the spin-diffusion length is much larger than the thickness of $\mathrm{N}$,

$$
\frac{\vec{\mu}_{s}(z)}{\mu_{s}^{0}} \stackrel{\lambda / d_{N} \gg 1}{=} \hat{m} \times(\hat{m} \times \hat{y})-\hat{y} \frac{2 z-d_{N}}{d_{N}},
$$

while spin current and magnetoresistance vanish. We can interpret this as multiple scattering of the spin current at the interfaces; the ISHE has both positive and negative charge current contributions that cancel each other.

\section{Spin Hall AHE}

Recent measurements in YIG|Pt display a small AHE-like signal on top of the ordinary Hall effect, i.e., a transverse voltage when the magnetization is normal to the film. ${ }^{31}$ As mentioned above, an imaginary part of the spin-mixing conductance $G_{i}$ can cause a spin Hall AHE (SHAHE).

The component of the spin accumulation $\mu_{s x}$

$$
\begin{aligned}
\frac{\mu_{s x}(z)}{\mu_{s}^{0}}= & \frac{2 \lambda}{\sigma} \frac{\cosh \frac{z-d_{N}}{\lambda}}{\sinh \frac{d_{N}}{\lambda}}\left[m_{x} m_{y} \operatorname{Re}-m_{z} \mathrm{Im}\right] \\
& \times \frac{\sigma G_{\uparrow \downarrow}}{\sigma+2 \lambda G_{\uparrow \downarrow} \operatorname{coth} \frac{d_{N}}{\lambda}}
\end{aligned}
$$

contains a contribution that scales with $m_{z}$ and contributes a charge current in the transverse $(\hat{y})$ direction

$$
\frac{j_{c, \text { trans }}^{(\mathrm{SHAHE})}(z)}{j_{c}^{0}}=-2 \lambda \theta_{\mathrm{SH}}^{2} m_{z} \frac{\sinh \frac{z-d_{N}}{\lambda}}{\sinh \frac{d_{N}}{\lambda}} \operatorname{Im} \frac{G_{\uparrow \downarrow} \tanh \frac{d_{N}}{2 \lambda}}{\sigma+2 \lambda G_{\uparrow \downarrow} \operatorname{coth} \frac{d_{N}}{\lambda}} .
$$

The transverse resistivity due to this current is

$$
\rho_{\text {trans }}^{\text {(SHAHE) }} \approx-\frac{\overline{j_{c, \text { trans }}^{\text {(SHAHE) }}} / E_{x}}{\sigma^{2}}=-\Delta \rho_{2} m_{z},
$$

where

$$
\begin{aligned}
\frac{\Delta \rho_{2}}{\rho} & \approx \frac{2 \lambda^{2} \theta_{\mathrm{SH}}^{2}}{d_{N}} \frac{\sigma G_{i} \tanh ^{2} \frac{d_{N}}{2 \lambda}}{\left(\sigma+2 \lambda G_{r} \operatorname{coth} \frac{d_{N}}{\lambda}\right)^{2}+\left(2 \lambda G_{i} \operatorname{coth} \frac{d_{N}}{\lambda}\right)^{2}} \\
& \approx \frac{2 \lambda^{2} \theta_{\mathrm{SH}}^{2}}{d_{N}} \frac{\sigma G_{i} \tanh ^{2} \frac{d_{N}}{2 \lambda}}{\left(\sigma+2 \lambda G_{r} \operatorname{coth} \frac{d_{N}}{\lambda}\right)^{2}} .
\end{aligned}
$$

\section{E. Comparison with experiments}

There are controversies about the values of the material parameters relevant for our theory, i.e., the spin-mixing conductance $G_{\uparrow \downarrow}$ of the N|F interface, as well as spin-flip diffusion length $\lambda$ and spin Hall angle $\theta_{\mathrm{SH}}$ in the normal metal.

Experimentally, Burrows et al. ${ }^{18}$ found for an Au|YIG interface with $G_{0}=e^{2} / h$,

$$
\frac{G_{r}^{\exp }}{G_{0}}=5.2 \times 10^{18} \mathrm{~m}^{-2} ; \quad G_{r}^{\exp }=2 \times 10^{14} \Omega^{-1} \mathrm{~m}^{-2} .
$$

On the theory side, the spin-mixing conductance from scattering theory for an insulator reads ${ }^{16}$

$$
\frac{G_{\uparrow \downarrow}}{G_{0}}=N_{\mathrm{Sh}}-\sum_{n} r_{n \uparrow}^{*} r_{n \downarrow}=N_{\mathrm{Sh}}-\sum_{n} e^{i\left(\delta_{n \downarrow}-\delta_{n \uparrow}\right)},
$$

where $r_{n \uparrow(\downarrow)}=e^{i \delta_{n \uparrow(\downarrow)}}$ is the reflection coefficient of an electron in the quantum channel $n$ on a unit area at the $\mathrm{N} \mid \mathrm{F}$ interface with unit modulus and phase $\delta_{n \uparrow(\downarrow)}$ for the majority (minority) spin, and $N_{\mathrm{Sh}}$ is the number of transport channels (per unit area) at the Fermi energy, i.e., $N_{\mathrm{Sh}}$ is the Sharvin conductance (for one spin). Therefore

$$
\frac{G_{r}}{G_{0}} \leqslant 2 N_{\mathrm{Sh}} ; \quad \frac{\left|G_{i}\right|}{G_{0}} \leqslant N_{\mathrm{Sh}} .
$$

Jia et al. ${ }^{17}$ computed Eq. (34) for a Ag|YIG interface by first principles. The average of different crystal interfaces

$$
G_{r}^{(0)}=2.3 \times 10^{14} \Omega^{-1} \mathrm{~m}^{-2},
$$

is quite close to the Sharvin conductance of silver $\left(N_{\mathrm{Sh}} G_{0} \approx\right.$ $4.5 \times 10^{14} \Omega^{-1} \mathrm{~m}^{-2}$ ).

For comparison with experiment we have to include the Schep drift correction: ${ }^{32}$

$$
\frac{1}{\tilde{G}_{r} / G_{0}}=\frac{1}{G_{r}^{(0)} / G_{0}}-\frac{1}{2 N_{\mathrm{Sh}}},
$$

which leads to

$$
\tilde{G}_{r} \approx 3.1 \times 10^{14} \Omega^{-1} \mathrm{~m}^{-2} .
$$

One should note that the mixing conductance of the Pt|YIG interface can then be estimated to be $\tilde{G}_{r} \approx 10^{15} \Omega^{-1} \mathrm{~m}^{-2}$ since the Pt conduction electron density and Sharvin conductance are higher than those of noble metals.

Using parameters $\rho=\sigma^{-1}=8.6 \times 10^{-7} \Omega \mathrm{m}, \quad d_{N}=$ $12 \mathrm{~nm}$, and $\lambda=1.5 \mathrm{~nm},{ }^{30}$ we see that the absorbed transverse spin currents with $G_{r}=\tilde{G}_{r}$ and $G_{r}=G_{r}^{\max }$ obtained from above for a $\mathrm{Ag} \mid \mathrm{YIG}$ interface are $44 \%$ and $70 \%$ of the value for a perfect spin sink $G_{r} \rightarrow \infty$, respectively. For a Pt|YIG interface this value should be even larger.

In order to compare our results with the observed SMR, we have to fill in or fit the parameters. The values of the spin-diffusion length and the spin Hall angle differ widely. ${ }^{30}$ In Fig. 3 we plot the SMR for three fixed values of $G_{r}$. We observe that the experiments can be explained by a sensible set of transport parameters $\left(G_{r}, \lambda, \theta_{\mathrm{SH}}\right)$ that somewhat differ for the two representative samples reported in Ref. 25. Generally, the SMR increases with a larger value of $G_{r}$ but decreases when $\lambda$ is getting longer. These features are in agreement with the discussion of the simple limits above. Sample 1 in Ref. 25 has a larger resistivity but a smaller SMR (ratio), implying a smaller spin Hall angle and/or smaller spindiffusion length. When we fix the spin Hall angle $\theta_{\mathrm{SH}}=0.06$ and the spin-mixing conductance $G_{r}=5 \times 10^{14} \Omega^{-1} \mathrm{~m}^{-2}$, the corresponding estimated spin-diffusion lengths of samples 1 and 2 are $\lambda_{1} \approx 1.5 \mathrm{~nm}$ and $\lambda_{2} \approx 3.5 \mathrm{~nm}$, respectively.

Finally we discuss the AHE equivalent (SHAHE). From experiments $\Delta \rho_{2} / \rho \approx 1.5 \times 10^{-5}$ for $\rho=4.1 \times 10^{-7} \Omega \mathrm{m}$ and $d_{N}=7 \mathrm{~nm}^{31}$ Choosing $\theta_{\mathrm{SH}}=0.05, \lambda=1.5 \mathrm{~nm}$, and $G_{r}=5 \times 10^{14} \Omega^{-1} \mathrm{~m}^{-2}$, we would need a $G_{i}=6.2 \times$ $10^{13} \Omega^{-1} \mathrm{~m}^{-2}$ to explain experiments, a number that is supported by first-principles calculations. ${ }^{17}$

\section{SPIN VALVES}

In this section we discuss $\mathrm{F}(\hat{m})|\mathrm{N}| \mathrm{F}\left(\hat{m}^{\prime}\right)$ spin valves fabricated from magnetic insulators with magnetization directions 

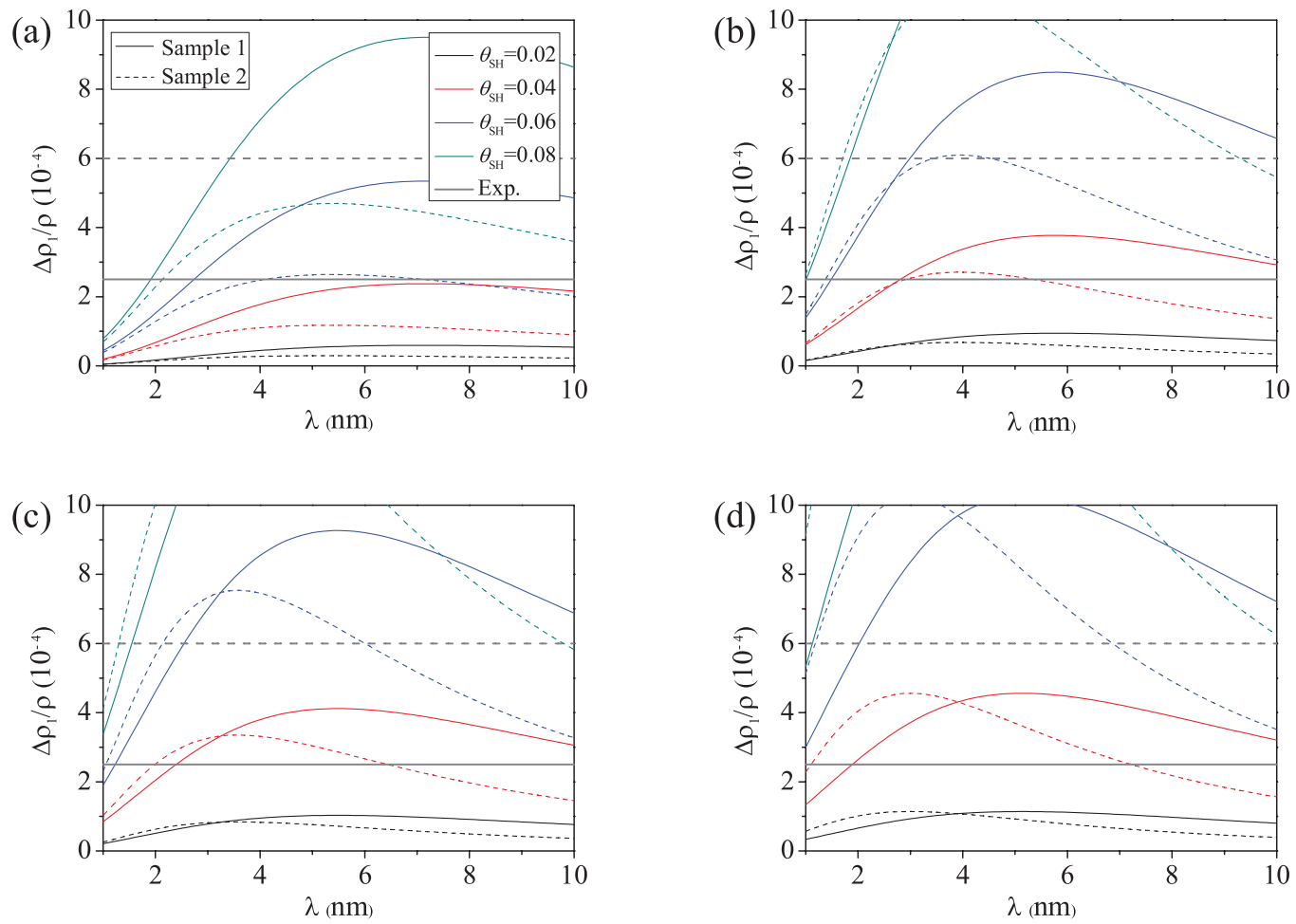

FIG. 3. (Color online) Calculated $\Delta \rho_{1} / \rho$ as a function of $\lambda$ for different spin Hall angles $\theta_{\mathrm{SH}}$ with (a) $G_{r}=1 \times 10^{14} \Omega^{-1} \mathrm{~m}^{-2}$, (b) $G_{r}=5 \times 10^{14} \Omega^{-1} \mathrm{~m}^{-2}$, (c) $G_{r}=10 \times 10^{14} \Omega^{-1} \mathrm{~m}^{-2}$, and (d) the ideal limit $G_{r} \gg \sigma /(2 \lambda)$. The Pt layers are $12 \mathrm{~nm}$ thick with resistivity $8.6 \times 10^{-7} \Omega \mathrm{m}$ (sample 1 , solid curve) and $7 \mathrm{~nm}$ thick with resistivity $4.1 \times 10^{-7} \Omega \mathrm{m}$ (sample 2, dashed curve). Experimental results are shown as horizontal lines for comparison (Ref. 25).

$\hat{m}$ and $\hat{m}^{\prime}$. The general angle dependence for independent rotations of $\hat{m}$ and $\hat{m}^{\prime}$ is straightforward but tedious. We discuss in the following two representative configurations in which the two magnetizations are parallel and perpendicular to each other. We disregard in the following the effective field due to $G_{i}$ such that the parallel and antiparallel configurations $\hat{m}= \pm \hat{m}^{\prime}$ are equivalent. Moreover, we limit the discussion to the simple case of two identical $\mathrm{F} \mid \mathrm{N}$ and $\mathrm{N} \mid \mathrm{F}$ interfaces, i.e., the spin-mixing conductances at both interfaces are the same.

\section{A. Parallel configuration $\left(\hat{\boldsymbol{m}} \cdot \hat{\boldsymbol{m}}^{\prime}= \pm \mathbf{1}\right)$}

When the magnetizations are aligned in parallel or antiparallel configuration, the boundary condition $\vec{j}_{s}^{(z)}\left(d_{N}\right)=$ $-\vec{j}_{s}^{(\mathrm{F})}$ applies. We proceed as in Sec. III to obtain the spin accumulation

$$
\frac{\vec{\mu}_{s}}{\mu_{s}^{0}}=-\left[\hat{y}+\hat{m} \times(\hat{m} \times \hat{y}) \frac{2 \lambda G_{r} \tanh \frac{d_{N}}{2 \lambda}}{\sigma+2 \lambda G_{r} \tanh \frac{d_{N}}{2 \lambda}}\right] \frac{\sinh \frac{2 z-d_{N}}{2 \lambda}}{\sinh \frac{d_{N}}{2 \lambda}},
$$

and the spin current

$$
\begin{aligned}
\frac{\vec{j}_{s}^{z}}{j_{s 0}^{\mathrm{SH}}}= & \hat{y}\left(\frac{\cosh \frac{2 z-d_{N}}{2 \lambda}}{\cosh \frac{d_{N}}{2 \lambda}}-1\right)+\hat{m} \times(\hat{m} \times \hat{y}) \\
& \times \frac{2 \lambda G_{r} \tanh \frac{d_{N}}{2 \lambda}}{\sigma+2 \lambda G_{r} \tanh \frac{d_{N}}{2 \lambda}} \frac{\cosh \frac{2 z-d_{N}}{2 \lambda}}{\cosh \frac{d_{N}}{2 \lambda}} .
\end{aligned}
$$

The spin currents at the bottom and top of $\mathrm{N}$ are absorbed as STTs and read

$$
\frac{\vec{j}_{s}^{z}(0)}{j_{s 0}^{\mathrm{SH}}}=\frac{\vec{j}_{s}^{z}\left(d_{N}\right)}{j_{s 0}^{\mathrm{SH}}}=\hat{m} \times(\hat{m} \times \hat{y}) \frac{2 \lambda G_{r} \tanh \frac{d_{N}}{2 \lambda}}{\sigma+2 \lambda G_{r} \tanh \frac{d_{N}}{2 \lambda}},
$$

leading to opposite STTs at the bottom $\left(\vec{\tau}_{\text {STT }}^{(\mathrm{B})}\right)$ and top $\left(\vec{\tau}_{\text {STT }}^{(\mathrm{T})}\right)$ ferromagnets

$$
\vec{\tau}_{\mathrm{STT}}^{(\mathrm{B})}=\frac{\hbar}{2 e} \vec{j}_{s}^{(z)}(0)=-\vec{\tau}_{\mathrm{STT}}^{(\mathrm{T})}
$$

since $\vec{j}_{s}^{(\mathrm{F})}(\hat{m})=\vec{j}_{s}^{z}(0)=\vec{j}_{s}^{z}\left(d_{N}\right)=-\vec{j}_{s}^{(\mathrm{F})}\left(\hat{m}^{\prime}\right)$.

The longitudinal and transverse (Hall) charge currents are

$$
\begin{aligned}
\frac{j_{c, \text { long }}}{j_{c}^{0}}= & 1+\theta_{\mathrm{SH}}^{2}\left[1-\left(1-m_{y}^{2}\right) \frac{2 \lambda G_{r} \tanh \frac{d_{N}}{2 \lambda}}{\sigma+2 \lambda G_{r} \tanh \frac{d_{N}}{2 \lambda}}\right] \\
& \times \frac{\cosh \frac{2 z-d_{N}}{2 \lambda}}{\cosh \frac{d_{N}}{2 \lambda}}, \\
\frac{j_{c, \text { trans }}}{j_{c}^{0}}= & -\theta_{\mathrm{SH}}^{2} m_{x} m_{y} \frac{2 \lambda G_{r} \tanh \frac{d_{N}}{2 \lambda}}{\sigma+2 \lambda G_{r} \tanh \frac{d_{N}}{2 \lambda}} \frac{\cosh \frac{2 z-d_{N}}{2 \lambda}}{\cosh \frac{d_{N}}{2 \lambda}}
\end{aligned}
$$

and the longitudinal and transverse resistivities read

$$
\begin{gathered}
\rho_{\text {long }}=\rho+\Delta \rho_{0}+\Delta \rho_{1}\left(1-m_{y}^{2}\right), \\
\rho_{\text {trans }}=\Delta \rho_{1} m_{x} m_{y},
\end{gathered}
$$




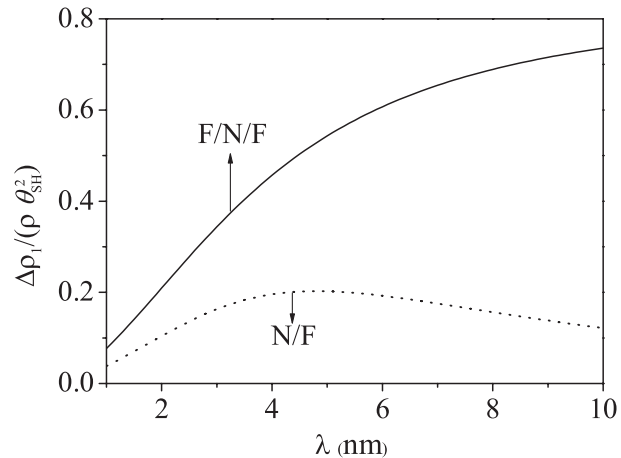

FIG. 4. Calculated $\Delta \rho_{1} /\left(\rho \theta_{\mathrm{SH}}^{2}\right)$ in an $\mathrm{F}|\mathrm{N}| \mathrm{F}$ spin valve as a function of spin-diffusion length $\lambda$ with $d_{N}=12 \mathrm{~nm}, G_{r}=5 \times$ $10^{14} \Omega^{-1} \mathrm{~m}^{-2}$, and $\rho=8.6 \times 10^{-7} \Omega \mathrm{m}$ chosen from sample 1 in Ref. 25. $\Delta \rho_{1} /\left(\rho \theta_{\mathrm{SH}}^{2}\right)$ in an $\mathrm{N} \mid \mathrm{F}$ bilayer is plotted as a dotted line for comparison.

where

$$
\begin{gathered}
\frac{\Delta \rho_{0}}{\rho}=-\theta_{\mathrm{SH}}^{2} \frac{2 \lambda}{d_{N}} \tanh \frac{d_{N}}{2 \lambda}, \\
\frac{\Delta \rho_{1}}{\rho}=\frac{\theta_{\mathrm{SH}}^{2}}{d_{N}} \frac{4 \lambda^{2} G_{r} \tanh ^{2} \frac{d_{N}}{2 \lambda}}{\sigma+2 \lambda G_{r} \tanh \frac{d_{N}}{2 \lambda}} .
\end{gathered}
$$

Figure 4 shows $\Delta \rho_{1} /\left(\rho \theta_{\mathrm{SH}}^{2}\right)$ with respect to the spin-diffusion length in an $\mathrm{F}|\mathrm{N}| \mathrm{F}$ spin valve with parallel magnetization configuration. Compared to $\mathrm{N} \mid \mathrm{F}$ bilayers, the SMR in spin valves is larger and does not vanish in the limit of long spin-diffusion lengths.

\section{B. Limit $\lambda / d_{N} \gg 1$}

The spin accumulation for weak spin flip reads

$\frac{\vec{\mu}_{s}}{\mu_{s}^{0}} \stackrel{\lambda / d_{N} \gg 1}{=}-\left[\hat{y}+\frac{d_{N} G_{r}}{\sigma+d_{N} G_{r}} \hat{m} \times(\hat{m} \times \hat{y})\right] \frac{2 z-d_{N}}{d_{N}}$,

leading to the spin current

$$
\frac{\vec{j}_{s}^{z}}{j_{s 0}^{\mathrm{SH}}} \stackrel{\lambda / d_{N} \gg 1}{=} \frac{d_{N} G_{r}}{\sigma+d_{N} G_{r}} \hat{m} \times(\hat{m} \times \hat{y}) .
$$

In contrast to the bilayer, we find a finite SMR in this limit for spin valves:

$$
\begin{gathered}
\frac{j_{c, \text { long }}}{j_{c}^{0}} \stackrel{\lambda / d_{N} \gg 1}{=} 1+\theta_{\mathrm{SH}}^{2}\left[1-\frac{d_{N} G_{r}}{\sigma+d_{N} G_{r}}\left(1-m_{y}^{2}\right)\right] \\
\stackrel{G_{r} \gg \sigma / d_{N}}{=} 1+\theta_{\mathrm{SH}}^{2} m_{y}^{2}, \\
\frac{j_{c, \text { trans }}}{j_{c}^{0}} \stackrel{\lambda / d_{N} \gg 1}{=}-\theta_{\mathrm{SH}}^{2} \frac{d_{N} G_{r}}{\sigma+d_{N} G_{r}} m_{x} m_{y} \stackrel{G_{r} \gg \sigma / d_{N}}{=}-\theta_{\mathrm{SH}}^{2} m_{x} m_{y}
\end{gathered}
$$

or

$$
\begin{gathered}
\frac{\Delta \rho_{0}}{\rho}=-\theta_{\mathrm{SH}}^{2}, \\
\frac{\Delta \rho_{1}}{\rho}=\theta_{\mathrm{SH}}^{2} \frac{d_{N} G_{r}}{\sigma+d_{N} G_{r}} \stackrel{G_{r} \gg \sigma / d_{N}}{=} \theta_{\mathrm{SH}}^{2} .
\end{gathered}
$$

Here we find the maximum achievable SMR effects in metals with spin Hall angle $\theta_{\mathrm{SH}}$ by taking the limit of perfect spin current absorption. Clearly this requires spin valves with sufficiently thin spacer layers. We interpret these results in terms of spin angular momentum conservation: The finite SMR is achieved by using the ferromagnet as a spin sink that suppresses the back flow of spins and the ISHE. This process requires a source of angular momentum, which in bilayers can only be the lattice of the normal metal. Consequently, the SMR is suppressed in the $\mathrm{F} \mid \mathrm{N}$ system when spin flip is not allowed. In spin valves, however, the second ferromagnet layer can act as a spin current source, thereby allowing a finite SMR even in the absence of spin-flip scattering.

\section{Perpendicular configuration $\left(\hat{m} \cdot \hat{m}^{\prime}=\mathbf{0}\right)$}

We may consider two in-plane magnetizations $\hat{m}=$ $(\cos \alpha, \sin \alpha, 0)$ and $\hat{m}^{\prime}=(-\sin \alpha, \cos \alpha, 0)$, which are perpendicular to each other. When $\alpha=0$, the first layer maximally absorbs the SHE spin current, while $\hat{m}^{\prime}$ is completely reflecting, just as the vacuum interface in the bilayer. For general $\alpha$,

$$
\begin{aligned}
& \frac{\mu_{s x}(z)}{\mu_{s}^{0}}= \frac{2 \lambda G_{r}}{\sigma+2 \lambda G_{r} \operatorname{coth} \frac{d_{N}}{\lambda}} \\
& \times\left(\frac{\cosh \frac{z-d_{N}}{\lambda}}{\sinh \frac{d_{N}}{\lambda}}+\frac{\cosh \frac{z}{\lambda}}{\sinh \frac{d_{N}}{\lambda}}\right) \cos \alpha \sin \alpha, \\
& \frac{\mu_{s y}(z)}{\mu_{s}^{0}}=-\frac{\sinh \frac{2 z-d_{N}}{2 \lambda}}{\sinh \frac{d_{N}}{2 \lambda}}-\frac{2 \lambda G_{r}}{\sigma+2 \lambda G_{r} \operatorname{coth} \frac{d_{N}}{\lambda}} \\
& \times\left(\frac{\cosh \frac{z-d_{N}}{\lambda}}{\sinh \frac{d_{N}}{\lambda}} \cos ^{2} \alpha-\frac{\cosh \frac{z}{\lambda}}{\sinh \frac{d_{N}}{\lambda}} \sin ^{2} \alpha\right), \\
& \mu_{s z}(z)=0,
\end{aligned}
$$

which leads to the components of spin current normal to the interfaces

$$
\begin{aligned}
\frac{j_{s x}(z)}{j_{s 0}^{\mathrm{SH}}=} & -\frac{2 \lambda G_{r} \tanh \frac{d_{N}}{2 \lambda}}{\sigma+2 \lambda G_{r} \operatorname{coth} \frac{d_{N}}{\lambda}} \\
& \times\left(\frac{\sinh \frac{z-d_{N}}{\lambda}}{\sinh \frac{d_{N}}{\lambda}}+\frac{\sinh \frac{z}{\lambda}}{\sinh \frac{d_{N}}{\lambda}}\right) \cos \alpha \sin \alpha, \\
\frac{j_{s y}(z)}{j_{s 0}^{\mathrm{SH}}}= & \frac{\cosh \frac{2 z-d_{N}}{2 \lambda}-\cosh \frac{d_{N}}{2 \lambda}}{\cosh \frac{d_{N}}{2 \lambda}}+\frac{2 \lambda G_{r} \tanh \frac{d_{N}}{2 \lambda}}{\sigma+2 \lambda G_{r} \operatorname{coth} \frac{d_{N}}{\lambda}} \\
& \times\left(\frac{\sinh \frac{z-d_{N}}{\lambda}}{\sinh \frac{d_{N}}{\lambda}} \cos ^{2} \alpha-\frac{\sinh \frac{z}{\lambda}}{\sinh \frac{d_{N}}{\lambda}} \sin ^{2} \alpha\right) .
\end{aligned}
$$

The total current is the sum of those from the two ferromagnets at the top and bottom; in contrast to the parallel $\hat{m}= \pm \hat{m}^{\prime}$ configuration, they do not feel each other. We can extend the discussion from the previous section: The second $F$ can be a spin current source, and we can switch this source on by rotating the magnetization from perpendicular to (anti)parallel configuration.

The longitudinal and transverse electric currents read

$$
\begin{aligned}
\frac{j_{c, \text { long }}(z)}{j_{c}^{0}}= & 1+\theta_{\mathrm{SH}}^{2} \frac{\cosh \frac{2 z-d_{N}}{2 \lambda}}{\cosh \frac{d_{N}}{2 \lambda}}+\theta_{\mathrm{SH}}^{2} \frac{2 \lambda G_{r} \tanh \frac{d_{N}}{2 \lambda}}{\sigma+2 \lambda G_{r} \operatorname{coth} \frac{d_{N}}{\lambda}} \\
& \times\left(\frac{\sinh \frac{z-d_{N}}{\lambda}}{\sinh \frac{d_{N}}{\lambda}} \cos ^{2} \alpha-\frac{\sinh \frac{z}{\lambda}}{\sinh \frac{d_{N}}{\lambda}} \sin ^{2} \alpha\right),
\end{aligned}
$$




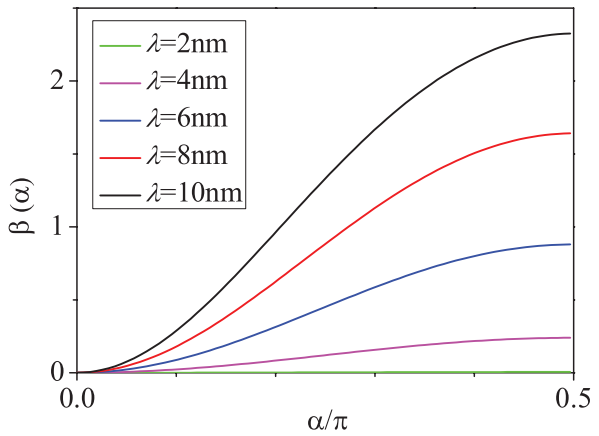

FIG. 5. (Color online) The ratio $\beta(\alpha)$ which characterize how $\vec{\tau}_{\text {STT }}^{(\mathrm{B})}$ changes with respect to the relative orientation between $\hat{m}$ and $\hat{m}^{\prime}$. We adopt the transport parameters $d_{N}=12 \mathrm{~nm}, \rho=8.6 \times 10^{-7} \Omega \mathrm{m}$, and $G_{r}=5 \times 10^{14} \Omega^{-1} \mathrm{~m}^{-2}$.

$$
\begin{aligned}
\frac{j_{c, \text { trans }}(z)}{j_{c}^{0}}= & \theta_{\mathrm{SH}}^{2} \frac{2 \lambda G_{r} \tanh \frac{d_{N}}{2 \lambda}}{\sigma+2 \lambda G_{r} \operatorname{coth} \frac{d_{N}}{\lambda}} \\
& \times\left(\frac{\sinh \frac{z-d_{N}}{\lambda}}{\sinh \frac{d_{N}}{\lambda}}+\frac{\sinh \frac{z}{\lambda}}{\sinh \frac{d_{N}}{\lambda}}\right) \cos \alpha \sin \alpha .
\end{aligned}
$$

Since the angle-dependent contributions vanish upon integration over $z$, there is no magnetoresistance in the perpendicular configuration.

\section{Controlling the spin-transfer torque}

Like the SMR, the STT at the N|F interface depends on the relative orientation between $\hat{m}$ and $\hat{m}^{\prime}$, too. We may pin $\hat{m}=\hat{x}$ and observe how the STT at the bottom magnet, $\vec{\tau}_{\text {STT }}^{(\mathrm{B})}\left(\hat{m}, \hat{m}^{\prime}\right)$, changes with rotating $\hat{m}^{\prime}=\hat{x} \cos \alpha+\hat{y} \sin \alpha$. Figure 5 displays the ratio $\beta$ defined as

$$
\beta(\alpha) \equiv \frac{\left|\vec{\tau}_{\mathrm{STT}}^{\mathrm{B})}(\hat{x}, \hat{x})-\vec{\tau}_{\mathrm{STT}}^{(\mathrm{B})}(\hat{x}, \hat{x} \cos \alpha+\hat{y} \sin \alpha)\right|}{\left|\vec{\tau}_{\mathrm{STT}}^{(\mathrm{B})}(\hat{x}, \hat{x})\right|},
$$

as a function of $\alpha$ for some spin-diffusion lengths. Only when $\lambda \ll d_{N}, \beta$ remains constant under rotation of $\hat{m}^{\prime}$. A larger spin-mixing conductance and smaller $d_{N}$ enhances the SMR as well as angle dependence of $\beta$. This modification of the STT should lead to complex dynamics of the spin valve in the presence of an applied current and will be the subject of a subsequent study.

\section{SUMMARY}

We developed a theory for the SMR in $\mathrm{N} \mid \mathrm{F}$ and $\mathrm{F}|\mathrm{N}| \mathrm{F}$ systems that takes into account the spin-orbit coupling in $\mathrm{N}$ as well as the spin transfer at the $\mathrm{N} \mid \mathrm{F}$ interface(s). In a $\mathrm{N} \mid \mathrm{F}$ bilayer system, the SMR requires spin flip in $\mathrm{N}$ and spin transfer at the $\mathrm{N} \mid \mathrm{F}$ interface. Our results explain the SMR measured in Ref. 25 both qualitatively and quantitatively with transport parameters that are consistent with other experiments. The degrees of spin accumulation in $\mathrm{N}$ that can be controlled by the magnetization direction is found to be very significant. In the presence of an imaginary part of the spin-mixing conductance $G_{i}$ we predicted an AHE-like signal (SHAHE). Such a signal was observed in Ref. 31 and can be explained with values of $G_{i}$ that agree with first-principles calculations. ${ }^{17}$ We furthermore analyzed $\mathrm{F}|\mathrm{N}| \mathrm{F}$ spin valves for parallel and perpendicular magnetization configurations. A maximal SMR $\sim \theta_{\mathrm{SH}}^{2}$ is found for a collinear magnetization configuration in the limit that the spin-diffusion length is much larger than the thickness of the normal spacer. The SMR vanishes when rotating the two magnetizations into a fixed perpendicular constellation. The SMR torques under applied currents in $\mathrm{N}$ are expected to lead to magnetization dynamics of $\mathrm{N} \mid \mathrm{F}$ and $\mathrm{F}|\mathrm{N}| \mathrm{F}$ structures.

\section{ACKNOWLEDGMENTS}

This work was supported by FOM (Stichting voor Fundamenteel Onderzoek der Materie), EU-ICT-7 "MACALO," the ICC-IMR, DFG Priority Programme 1538 "Spin-Caloric Transport" (GO 944/4), and KAKENHI (22540346).
${ }^{1}$ S. D. Bader and S. S. P. Parkin, Annu. Rev. Condens. Matter Phys. 1, 71 (2010).

${ }^{2}$ J. Sinova and I. Žutić, Nature Mater. 11, 368 (2012).

${ }^{3}$ For a review, see T. Jungwirth, J. Wunderlich, and K. Olejník, Nature Mater. 11, 382 (2012).

${ }^{4}$ K. Ando, S. Takahashi, K. Harii, K. Sasage, J. Ieda, S. Maekawa, and E. Saitoh, Phys. Rev. Lett. 101, 036601 (2008).

${ }^{5}$ I. M. Miron, K. Garello, G. Gaudin, P.-J. Zermatten, M. V. Costache, S. Auffret, S. Bandiera, B. Rodmacq, A. Schuhl, and P. Gambardella, Nature (London) 476, 189 (2011).

${ }^{6}$ L. Liu, C. F. Pai, Y. Li, H. W. Tseng, D. C. Ralph, and R. A. Buhrman, Science 336, 555 (2012).

${ }^{7}$ E. Saitoh, M. Ueda, H. Miyajima, and G. Tatara, Appl. Phys. Lett. 88, 182509 (2006).

${ }^{8}$ O. Mosendz, J. E. Pearson, F. Y. Fradin, G. E. W. Bauer, S. D. Bader, and A. Hoffmann, Phys. Rev. Lett. 104, 046601 (2010).
${ }^{9}$ O. Mosendz, V. Vlaminck, J. E. Pearson, F. Y. Fradin, G. E. W. Bauer, S. D. Bader, and A. Hoffmann, Phys. Rev. B 82, 214403 (2010).

${ }^{10}$ F. D. Czeschka, L. Dreher, M. S. Brandt, M. Weiler, M. Althammer, I.-M. Imort, G. Reiss, A. Thomas, W. Schoch, W. Limmer, H. Huebl, R. Gross, and S. T. B. Goennenwein, Phys. Lett. Rev. 107, 046601 (2011).

${ }^{11}$ K. Uchida, S. Takahashi, K. Harii, J. Ieda, W. Koshibae, K. Ando, S. Maekawa, and E. Saitoh, Nature (London) 455, 778 (2008).

${ }^{12}$ C. M. Jaworski, J. Yang, S. Mack, D. D. Awschalom, J. P. Heremans, and R. C. Myers, Nature Mater. 9, 898 (2010).

${ }^{13}$ K. Uchida, J. Xiao, H. Adachi, J. Ohe, S. Takahashi, J. Ieda, T. Ota, Y. Kajiwara, H. Umezawa, H. Kawai, G. E. W. Bauer, S. Maekawa, and E. Saitoh, Nature Mater. 9, 894 (2010).

${ }^{14}$ M. Weiler, M. Althammer, F. D. Czeschka, H. Huebl, M. S. Wagner, M. Opel, I.-M. Imort, G. Reiss, A. Thomas, R. Gross, and S. T. B. Goennenwein, Phys. Rev. Lett. 108, 106602 (2012). 
${ }^{15}$ Y. Kajiwara, K. Harii, S. Takahashi, J. Ohe, K. Uchida, M. Mizuguchi, H. Umezawa, H. Kawai, K. Ando, K. Takanashi, S. Maekawa, and E. Saitoh, Nature (London) 464, 262 (2010).

${ }^{16}$ A. Brataas, Yu. V. Nazarov, and G. E. W. Bauer, Phys. Rev. Lett. 84, 2481 (2000); Eur. Phys. J. B 22, 99 (2001).

${ }^{17}$ X. Jia, K. Liu, K. Xia, and G. E. W. Bauer, Europhys. Lett. 96, 17005 (2011).

${ }^{18}$ C. Burrowes, B. Heinrich, B. Kardasz, E. A. Montoya, E. Girt, Y. Sun, Y. Y. Song, and M. Wu, Appl. Phys. Lett. 100, 092403 (2012).

${ }^{19}$ N. W. Ashcroft and N. D. Mermin, Solid State Physics (Saunders, Philadelphia, 1976).

${ }^{20}$ T. R. McGuire and R. I. Potter, IEEE Trans. Magn. 11, 1018 (1975).

${ }^{21}$ D. A. Thompson, L. T. Romankiw, and A. F. Mayadas, IEEE Trans. Magn. 11, 1039 (1975).

${ }^{22}$ N. Nagaosa, J. Sinova, S. Onoda, A. H. MacDonald, and N. P. Ong, Rev. Mod. Phys. 82, 1539 (2010).

${ }^{23}$ A. Fert, Rev. Mod. Phys. 80, 1517 (2008).
${ }^{24}$ S. Y. Huang, X. Fan, D. Qu, Y. P. Chen, W. G. Wang, J. Wu, T. Y. Chen, J. Q. Xiao, and C. L. Chien, Phys. Rev. Lett. 109, 107204 (2012).

${ }^{25}$ H. Nakayama, M. Althammer, Y.-T. Chen, K. Uchida, Y. Kajiwara, D. Kikuchi, T. Ohtani, S. Geprägs, M. Opel, S. Takahashi, R. Gross, G. E. W. Bauer, S. T. B. Goennenwein, and E. Saitoh, Phys. Rev. Lett. (in press).

${ }^{26}$ N. Vlietstra, J. Shan, V. Castel, J. Ben Youssef, and B. J. van Wees, arXiv:1301.3266.

${ }^{27}$ M. I. Dyakonov, Phys. Rev. Lett. 99, 126601 (2007).

${ }^{28}$ S. Takahashi, H. Imamura, and S. Maekawa, in Concepts in Spin Electronics, edited by S. Maekawa (Oxford University Press, UK, 2006), pp. 343-370.

${ }^{29}$ P. C. van Son, H. van Kempen, and P. Wyder, Phys. Rev. Lett. 58, 2271 (1987).

${ }^{30}$ L. Liu, R. A. Buhrman, and D. C. Ralph, arXiv:1111.3702.

${ }^{31}$ M. Althammer et al., Phys. Rev. B (submitted).

${ }^{32}$ K. M. Schep, J. B. A. N. van Hoof, P. J. Kelly, G. E. W. Bauer, and J. E. Inglesfield, Phys. Rev. B 56, 10805 (1997). 